\title{
Multiple-resolution divide and conquer neural networks for large-scale TSP-like energy minimization problems
}

\author{
Steven Noel and Harold Szu \\ Naval Surface Warfare Center Dahlgren Division, Dahlgren, VA
}

\begin{abstract}
Here we describe a multiple-resolution divide-andconquer ANN approach for large-scale constrained optimization problems like the TSP. The goal of the approach is to divide a problem into sub-problems, solve them with parallel ANNs, and then combine the resulting sub-solutions. The divide-and-conquer approach is based on a mathematical principle of orthogonal division errors, which provides the criteria for optimal problem division. The resulting division minimizes the cross-correlation among sub-problems, and hence the necessary communication among them. It therefore provides a minimum-communication allocation of sub-problems to parallel processors. Moreover, the divide and conquer can be done recursively, so that it occurs at all resolutions of the data. We show how wavelets can perform the necessary multiple-resolution data clustering. The divide-and-conquer approach is particularly effective for large-scale fractal data, which exhibits clustering over a large number of resolutions.
\end{abstract}

\section{Introduction}

Historically, the application of ANNs to constrained optimization was pioneered by Hopfield and Tank [1] in the mid-80s. Unfortunately, the initial excitement over their success for the TSP was somewhat dampened when Wilson and Pawley [2] pointed out stability problems. However, it was later determined that the problems were not intrinsic to ANNs, but rather were caused by the particular choices of network constraints. Necessary and sufficient conditions were found by Szu [3] which ensure that the ground states of the permutation matrix computation are valid TSP tours.

The principle of divide and conquer is a very obvious and frequently successful approach to problem solving. The idea is to divide a large problem into pieces, solve the divided sub-problems separately, then combine the sub-solutions into a full solution. ANNs have implicit, adaptive forms of divide and conquer. At the beginning of the optimization process, the more global decisions are made. Over time, the global parts of the solution tend to become fixed, and only more local decisions are made. The change from global to local optimization corresponds to an increase in the level of divide and conquer. Our goal is to improve the performance of ANNs for constrained optimization by adding a more explicit form of divide and conquer.

The architecture for such an explicit ANN divide and conquer is shown in Figure 1. A division algorithm splits the problem into sub-problems and allocates them among separate networks running in parallel. A recombination algorithm then combines the sub-solutions. This could even be generalized to allow the parallel ANNs to be replaced with other types of optimization algorithms, for example simulated annealing [4][5], genetic algorithms [6], or the elastic net method [7].

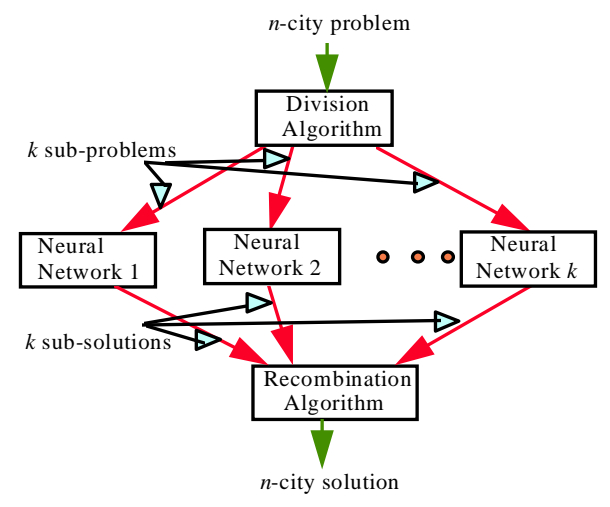

\section{Figure 1. Architecture for divide and conquer ANN}

The mathematical necessity of a divide and conquer approach for the TSP could perhaps be traced back to the well-known "curse of dimensionality." This is seen in the reduction of relative phase space for valid tours as the number of cities increases. The number of possible solutions $S$ for an $n$-city TSP with 
symmetric distances is

$$
S=\frac{n !}{2 n} \text {. }
$$

Here $n$ ! is the number of permutations of $n$ cities, the division by $n$ is because a tour can start and end at any city, and the division by 2 is because of distance symmetry between pairs of cities. The TSP permutation matrix for $n$ cities has $n^{2}$ elements, each of which can take on 2 possible values, so that the total phase space $P$ of the permutation matrix is

$$
P=2^{\mathrm{n}^{2}} \text {. }
$$

The relative phase space $S / P$ for large $n$ is then

$$
\begin{aligned}
\lim _{n \rightarrow \infty} \frac{S}{P} & =\lim _{n \rightarrow \infty} \frac{n ! /(2 n)}{2^{n^{2}}} \\
& \approx \lim _{n \rightarrow \infty} e^{-n}=0,
\end{aligned}
$$

Thus the probability of the permutation matrix being in a valid TSP state thus becomes vanishingly small for large $n$.

Because of the nonlinearity of the TSP, a divide and conquer must be carefully implemented to maintain solution quality. Here an important mathematical principle applies - the "Theorem of Orthogonal Division Errors" [8]. It is based on an isomorphism between the lossless division of a TSP and the lossless division of a least-mean-square energy problem. The division of a TSP into 2 sub-problems is represented by

$$
\min |\mathbf{V}|^{2}=\min |\mathbf{A}|^{2}+\min |\mathbf{B}|^{2}+2 \min (\mathbf{A}, \mathbf{B}) .
$$

$\mathbf{V}$ is the ensemble of displacement vectors for all possible TSP tours, and $\mathbf{A}$ and $\mathbf{B}$ are the displacement vector ensembles for the 2 sub-problems. These displacement vector ensembles are analogous to the kinetic energy vector ensembles for least-meansquare energy problems. The theorem states that the division becomes lossless when the inner product $(\mathbf{A}, \mathbf{B})$ vanishes, so that the sum of the sub-solutions is the full solution. The inner product represents the cross-correlation between the 2 sub-problems. While in general it is non-trivial and often impossible to completely eliminate the cross-correlation, the goal is to at least minimize it. This theorem is valid for any space in which the triangle inequality holds, that is for any metric space.

Divide and conquer has a potential for reducing problem complexity that could yield a far greater speedup beyond parallelism. This makes it attractive even when implemented serially. Of course, additional computation is needed for the division and recombination operations, but in general this should be relatively insignificant. Consider the simple example of an $n$-city TSP divided into $k$ equal parts. The original number of possible solutions $S$ is given by (1). Since we are now solving $k$ independent subproblems, the number of possible solutions with divide and conquer $S_{\mathrm{DC}}$ is

$$
S_{\mathrm{DC}}=k\left[\frac{(n / k) !}{2 n / k}\right]=\frac{k^{2}(n / k) !}{2 n} .
$$

The relative reduction in problem complexity $S_{\mathrm{DC}} / S$ is then

$$
\frac{S_{\mathrm{DC}}}{S}=\frac{k^{2}(n / k) !}{n !} .
$$

As a numerical example, a 100-city TSP divided into 10 sub problems has the relative complexity reduction

$$
\frac{S_{\mathrm{DC}}}{S}=\frac{10^{2} \cdot 10 !}{100 !} \approx 10^{150},
$$

which is a very large improvement.

The relative complexity reduction is even more dramatic when the divide and conquer is done recursively. For example, consider a $10^{6}$-city problem, divided recursively into 10 clusters of equal size at each level, through 6 levels. We now have

$$
\frac{S_{\mathrm{DC}}}{\mathrm{S}}=\frac{\left(10^{5}\right)^{2}(10 !)}{10^{6} !} \approx \frac{10^{16}}{10^{6} !} .
$$

The number $10^{6}$ ! is too large to compute digitally. We therefore use an approximation based on a series expansion of the gamma funtion for large $m$ :

$$
10^{m} ! \approx\left(10^{m-1}\right)^{10^{m}} 10^{m / 2}=10^{(m-1) 10^{m}+m / 2} .
$$

The relative complexity reduction is then

$$
\frac{S_{\mathrm{DC}}}{S} \approx \frac{10^{6}}{10^{5 \times 10^{6}+3}},
$$

so that the problem complexity is incredibly reduced.

When implemented serially, ANNs represent a compromise, in terms of complexity and optimality, between enumerative and heuristic optimization algorithms. However, they also provide opportunities for parallelism. Unfortunately, the typical parallel computer has processors that operate synchronously under a clock cycle. Communication costs among processors thus result in a diminishing return on the parallel speedup as the number of processors sufficiently increases. The largest possible speedup has been estimated at 3 orders of magnitude above the serial limit [3]. There exist other massively parallel, 6th-generation ANNs [9] which are asynchronous, having neither lock step communication nor clock cycles. These operate at speeds 3 orders of magnitude beyond conventional parallel machines. Attempts at 
explaining the speedup for asynchronous communication led to the Theorem of Orthogonal Division Errors. The theorem essentially describes an allocation of sub-problems that minimizes the necessary communication among processors.

\section{Recursive divide and conquer}

The theorem of orthogonal division errors gives no specific algorithm for dividing a TSP, only the mathematical criterion for minimizing the crosscorrelation between the sub-problems. We therefore examine the idea of clustering as the division mechanism. Intuitively, if the clusters are sufficiently well separated, points from one cluster have minimal correlation with those from another. In fact, it appears that humans perform some sort of clusterbased divide and conquer when they solve TSPs by hand. Moreover, we are strongly interested in data with a fractal distribution. Since this type of data has clusters at all scales, we expect to be able to perform the clustering recursively. Such a recursive or nested clustering is shown in Figure 2

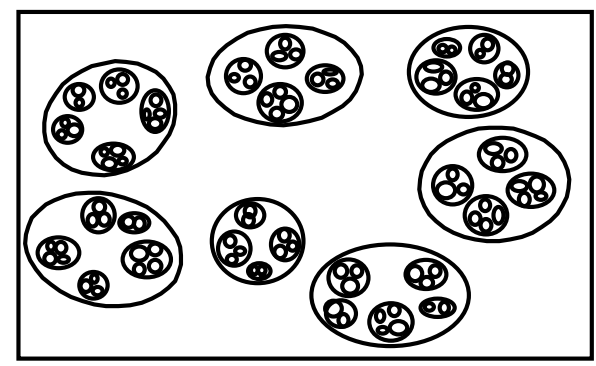

Figure 2. Recursive clustering of cities

Once the TSP is divided into sub-problems, we need to find optimal solutions for them, and then find a way to combine them. As a human might do when solving a TSP, we start with the highest-level (largest) clusters first. We use some appropriate distance measure for this highest-level optimization, such as distances between cluster centers. This is shown in the upper left of Figure 3. We now know how the highest-level clusters are linked for a near-optimal tour. We then identify boundary clusters at the next lowest level to actually link the highest-level clusters. These could be the closest pairs of sub-clusters between 2 linked clusters. The upper right of Figure 3 shows the linking of boundary sub-clusters, which are labeled B.

Within each high-level cluster, we can now find the optimal path through the lower-level clusters that begins and ends at the boundary ones. This is shown in the lower left of Figure 3. Note that the intracluster paths are Hamiltonian paths and not Hamiltonian loops. We now form the union of intercluster and intra-cluster links to get the near-optimal tour at the second level of clustering, as shown in the lower right of Figure 3. Note that the higher-level optimal paths constrain the lower-level ones through the boundary sub-clusters. This is desirable since the higher-level paths contribute more to solution optimality.

We can continue this process recursively until we reach lowest-level clusters of a manageable size. This top-down recursion could also be considered a form of data compression, since if given a communication medium of limited bandwidth, the most important parts of the solution could be transmitted first. Intuitively, the optimality of recursive divide and conquer would depend on the degree of clustering inherent in the data at the various levels.

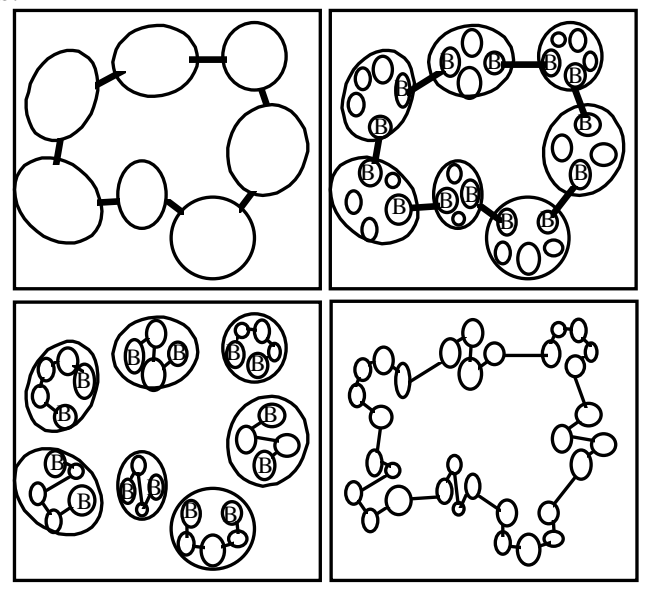

Figure 3. Recursive divide and conquer

\section{Recursive divide and conquer implies multiple resolutions}

We now consider a more illustrative example of recursive divide and conquer. In this example, a manager of the Burger World restaurant chain has been given a daunting task: visit each of the restaurants in the continental US to get a first-hand look at operations. Our manager wants to plan the shortest possible itinerary. Unfortunately, he soon realizes that finding the shortest itinerary, given the thousands of Burger World restaurants in the country, could take longer than the actual trip. Eventually he gets a brilliant insight: "Wait a minute! In planning 
the trip, I can first just find the best route between the biggest groups of restaurants in the country. There aren't all that many of the biggest groups. Once I do that, I can zoom in on each of the groups and plan my route within them. After all, I don't need to worry about locations in Los Angeles as I'm planning the route for New York. I'll bet there are also groups of restaurants within each big group, so I can do the same thing for them. In fact, I should be able to keep doing that until my final groups are a manageable size. This will be easy! Too bad I still have to make the stinking trip." The manager is implementing a multiple-level divide-and-conquer strategy. He begins by forming clusters and finding the optimal tour at the highest level by looking a world map. This map has the largest scale and hence the lowest resolution, which he needs in order for the larger clusters to appear essentially as points. He then uses maps of increasing resolution as he moves to lower levels. We can easily see that multiple-level clustering implies multiple resolutions.

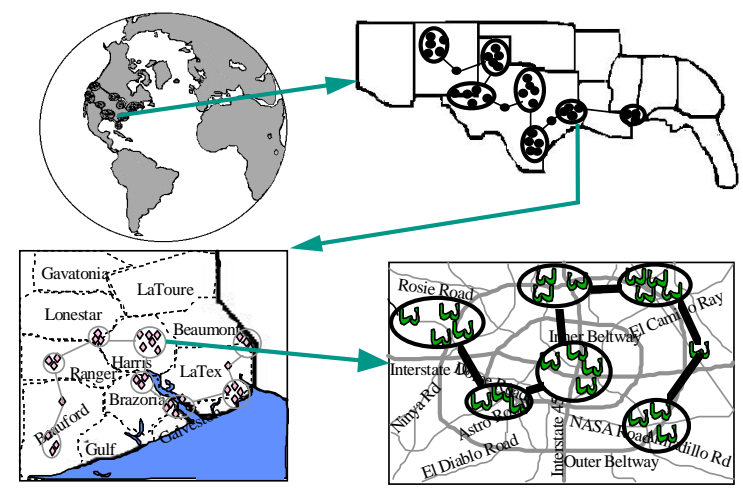

Figure 4. Sequence of maps for optimal tour of restaurants

Recent work by Noel and Szu has shown that the necessary multiple-resolution clustering can be accomplished with wavelets [10]. This is not surprising, since wavelets are the natural tool for handling multiple resolutions. The idea is to map TSP city coordinates to a digital image, then apply Mallat's multiresolution analysis (MRA) [11]. A threshold scheme is then applied to form discrete clusters of cities from continuous MRA representations. Figure 5 shows such a multiple-resolution clustering using the Daubechies s8 "symmlet" [12] as the analyzing wavelet. The darker regions indicate higherresolution clusters.

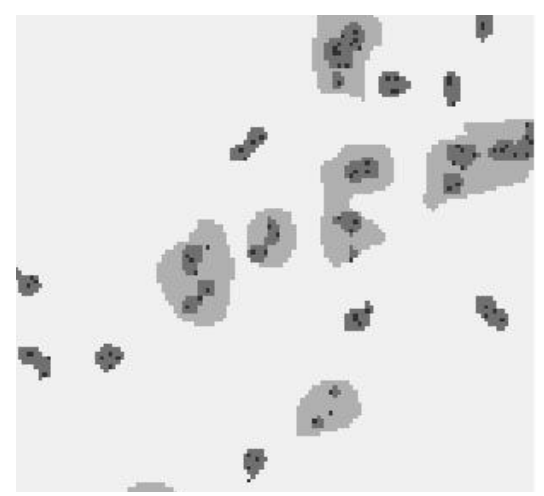

\section{Figure 5. Cluster formation by applying thresholds to MRA (using Daubechies s8 wavelet)}

\section{Optimality of multiple-resolution divide and conquer}

We now show how the optimality of divide and conquer depends on the degree of inherent data clustering at various resolutions. As our example we use the Sierpinski-type triangle shown in the left-hand side of Figure 6 . The triangle vertices are the TSP cities, which we cluster recursively over 2 levels, as shown in the right-hand side of Figure 6 . We choose the parameters $r>1$ and $s>1$ to vary the degree of clustering.

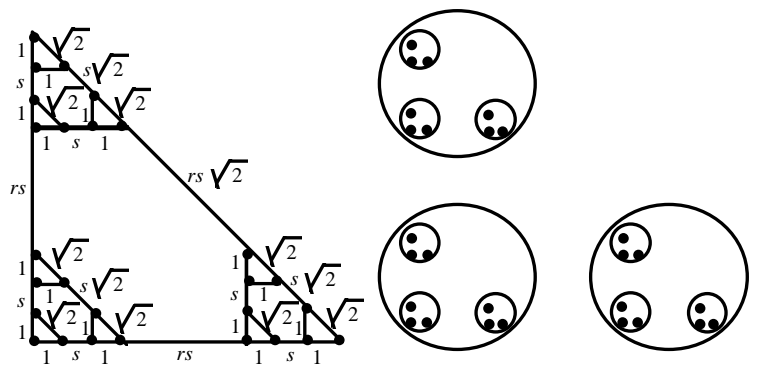

\section{Figure 6. Distances and clustering for recursive triangles problem}

The divide-and-conquer solution is shown in the left-hand side of Figure 7. This solution is suboptimal. The optimal solution appears in the center of Figure 7. We can write the divide-and-conquer tour length $l_{\mathrm{DC}}(r, s)$ and the optimal tour length $l_{\mathrm{opt}}(r, s)$ as

$$
l_{\mathrm{DC}}(r, s)=(2+\sqrt{2}) r s+2(2+\sqrt{2}) r+6 \sqrt{2}+12
$$




$$
\begin{aligned}
l_{\text {opt }}(r, s)= & r s \sqrt{2}+2 \sqrt{1+r^{2} s^{2}}+2 \sqrt{1+r s}+ \\
& 2(1+\sqrt{2}) r+2 \sqrt{2}+16 .
\end{aligned}
$$

Since

$$
\lim _{r, s \rightarrow \infty} \frac{l_{\mathrm{DC}}}{l_{\mathrm{opt}}}=1,
$$

the divide and conquer solution converges to the optimal one as the cluster separations increase. In fact, even for the relatively small separations $r=2$ and $r s=4$ the divide and conquer tour is only $2 \%$ longer than optimal. The right-hand side of Figure 7 shows the ratio $l_{\mathrm{DC}} / l_{\mathrm{opt}}$ for $r=s$.
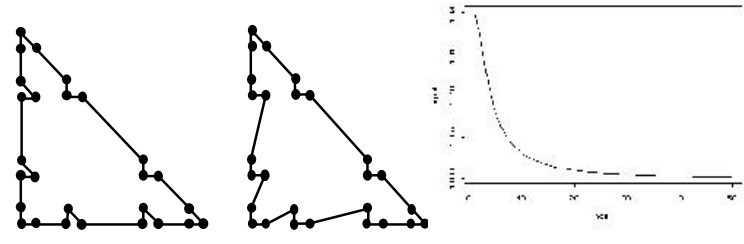

Figure 7. Divide and conquer tour, optimal tour, and their ratio as a function of $r=s$

\section{Computational divide and conquer}

We next do a computational implementation of a TSP divide and conquer. We perform a single level of clustering with the k-means algorithm [13], with the number of clusters $k$ chosen to be 9 . For the initial clustering required by the k-means algorithm we use hierarchical clusters [13] with average linkage. The TSP city coordinates, labeled according to the k-means clusters, is shown in Figure 8. To find both the intercluster and intra-cluster optimal tours, we use a genetic algorithm [6] written in $\mathrm{C}$ language. It is the non-overlapping generational model [6] with population size 100. Parent selection is done with stochastic universal sampling [14], a form of proportional selection. Each generation, population fitnesses are scaled linearly to lie between zero and one. The algorithm has permutation encoding [6], with order crossover [15] and pair-swapping mutation, and operator probabilities of 0.4 and 0.002 . For points within clusters, the algorithm was modified to find the optimal path (versus loop) which was constrained by the cluster boundary points. To find the boundary points, we use a simple program that compares distances between all pairs of points between each of the cluster pairs dictated by the optimal inter-cluster tour, and retains the smallest.
The resulting divide-and-conquer solution is shown in the right-hand side of Figure 8. Note that parts of the solution are sub-optimal (within clusters 3 and 6). This is caused entirely by the genetic algorithm, and is independent of the divide-andconquer approach. In fact it reminds us of the desirability of the divide-and-conquer approach. If the same genetic algorithm was used for the full problem, without the divide and conquer, the convergence would be extremely slow, and the resulting solutions would be much worse.
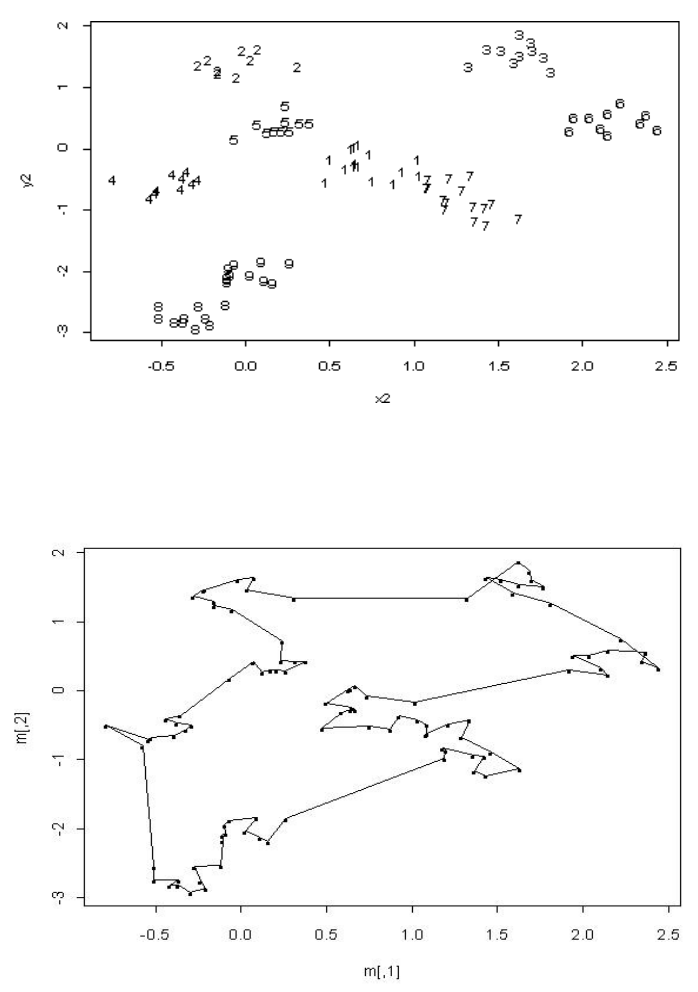

Figure 8. k-means clustering, and divide-and-conquer solution (suboptimality is independent of divide and conquer)

\section{Conclusions and remarks}

We have examined a multiple-resolution divide-and-conquer approach to combinatorial optimization. The approach relies on a mathematical principle of minimizing the cross-correlation between the divided sub-problems, which is analogous to the division of least-mean-square kinetic energy problems. The need to minimize sub-problem cross-correlation 
leads to the idea of multiple-level clustering. We showed how multiple-level clustering implies multiple resolutions, and how wavelets can form the necessary clusters. We also analyzed the relative optimality of a TSP divide and conquer to demonstrate how solution quality improves with increased cluster separation. Finally, we gave computational results for a TSP divide and conquer.

In closing, we would like to stress the fundamental importance of effective approaches to constrained optimization problems like the TSP. These rank among the most computationally difficult of all known problems, and strike at the core of computability. Such problems also frequently arise in applications, for example in such diverse areas as processor allocation, postal scheduling, product distribution, and military target assignment.

\section{References}

[1] J. Hopfield, D. Tank, "Neural Computation of Decisions in Optimization Problems," Biological Cybernetics, Vol. 55, pp. 141-152, 1985.

[2] G. Wilson, G. Pawley, "On the Stability of the Traveling Salesman Problem Algorithm of Hopfield and Tank," Biological Cybernetics, Vol. 58, pp. 63-70, 1988.

[3] H. Szu, "Fast TSP Algorithm Based on Binary Neuron Output and Analog Neuron Input Using the ZeroDiagonal Interconnect Matrix and Necessary and Sufficient Constraints of the Permutation Matrix," Proc. IEEE ICNN, San Diego, 1988.

[4] S. Kirkpatrick, C. Gelatt, M. Vecchi, "Optimization by Simulated Annealing,” Science 220, pp. 671-680, 1983.

[5] H. Szu, "Fast Simulated Annealing,", Snowbird Utah Conf. Am. Inst. Phys. (Denker ed.) 15, pp. 420-425, 1987.

[6] D. E. Goldberg, Genetic Algorithms in Search, Optimization, and Machine Learning, AddisonWesley, 1989.

[7] R. Durbin, D. Willshaw, "An Analogue Approach to the TSP Using an Elastic Net Method, Nature 326, 1987.

[8] H. Szu, S. Foo, "Divide and Conquer Orthogonality Principle for Parallel Optimizations in the TSP," Neurocomputing 7, Elsevier, pp. 1-13, 1994.

[9] H. Szu, "Sixth Generation Computing Architectures," Learning and Recognition: A Modern Approach (K. Zhao et al eds.), World Scientific, pp. 59-65, 1988.

[10] S. Noel, H. Szu, "Multiple Resolution Clustering for Recursive Divide and Conquer," Proc. SPIE Wavelet Apps. IV, Orlando, FL, 1997.

[11] S. Mallat, "A Theory for Multiresolution Signal Decomposition: The Wavelet Representation,"
IEEE Trans. Patt. Anal. and Mach. Intel., Vol. 11, No. 7, pp. 674-693, 1989.

[12] I. Daubechies, Ten Lectures on Wavelets, SIAM, 1992.

[13] J. Hartigan, Clustering Algorithms, John Wiley and Sons, 1975.

[14] J. E. Baker, "Reducing Bias and Inefficiency in the Selection Algorithm," Proc. Second Int. Conf. On Genetic Algorithms, Lawrence Erlbaum, pp. 14-21, 1987.

[15] I. M. Oliver, D. J. Smith, J. R. C. Holland, "A Study of Permutation Crossover Operators on the Traveling Salesman Problem," Proc. Second Intl. Conf. On Genetic Algorithms, Lawrence Erlbaum, pp. 224230, 1987.

[16] C. Papadimitriou, Combinatorial Optimization: Algorithms and Complexity, Prentice-Hall.

[17] E. L. Lawler, et al (eds.), The Traveling Salesman Problem, John Wiley \& Sons, 1985.

[18] S. Lin, B. Kernighan, "An Effective Heuristic Algorithm for the Travelling-Salesman Problem," Operations Research, Vol. 21, pp. 498-516, 1973. 